\title{
A Practical Servomotor Project: Combining the Web with Simulation Tools to Solidify Concepts in Undergraduate Control Education
}

\section{Malgorzata S. Zywno}

Ryerson University

\section{Diane C. Kennedy}

Ryerson University

Zywno, M. S., \& Kennedy, D. C. (1998). A practical servomotor project:

Combining the Web with simulation tools to solidify concepts in undergraduate control education. Proceedings of the 1998 American Control Conference. ACC (IEEE Cat. No.98CH36207), 2, 1309-1313.

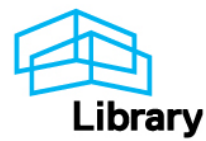




\title{
A Practical Servomotor Project: Combining the Web with Simulation Tools to Solidify Concepts in Undergraduate Control Education
}

\author{
Malgorzata S. Zywno and Diane C. Kennedy \\ Department of Electrical and Computer Engineering, Ryerson Polytechnic University \\ 350 Victoria Street, Toronto, Ontario, M5B 2K3, CANADA \\ E-mail:gosha@ee.ryerson.ca,dkennedy@ee.ryerson.ca
}

\begin{abstract}
Current technology enables the lecturer to use computer tools to enhance the conceptualisation of lecture material. This can be especially useful in an engineering curriculum, as the course material can be rendered less abstract through visual illustration of difficult mathematical concepts.

In this paper, we present an example of a multimedia enhanced course in linear control theory; taught by authors at Ryerson Polytechnic University (Toronto, Canada). In our implementation of the course, we combine software simulations of practical systems to illustrate control theory concepts with extensive on-line course notes to assist with comprehension.

Followup analysis shows that the use of the WWW and computer tools to enhance the learning process leads to increased enthusiasm, comprehension, and information retention. A review of the process required to create the technology enabled learning environment shows that initially there is a great deal of work involved. However, we conclude that the effort is justified by allowing a positive qualitative change in the way we educate engineering students.

Key Words- Control Engineering Education; Multimedia Applications; World Wide Web.
\end{abstract}

\section{Introduction}

One of the required courses for undergraduate students of Electrical and Computer Engineering is the introductory course in classic linear control theory, typically taught in the third year of study. Embedded in this course are mathematical concepts of control theory, which are often difficult to grasp. For example, topics such as convolution, contour mapping, or the effects of poles and zeros on system response are sometimes easier to understand if we exploit an engineer's inherent preference to use visualisation rather than to think in an abstract fashion. In fact, classical approaches to teaching often include the use of chalkboard diagrams

$0-7803-4530-4 / 98 \$ 10.00$ (c) 1998 AACC and graphs in conjunction with lecture notes, to assist in explaining the mathematical intricacies of control theory via visualisation of the concepts. Finally, to improve comprehension and retention, tutorials, assignments, and associated laboratory experiments round out the typical course delivery.

In Ryerson's curriculum, the laboratory component tends to be quite demanding. Furthermore, it is important to note that this is the first time that the student is required to deal with a more complex experiment than can be resolved in a single standard three hour lab session. The laboratory experiments are also used to implicitly provide an additional benefit of laying the foundation for future practice of control engineering in industry.

In our work, we adopt an approach which combines multimedia tools with some of the components of the traditional course curriculum to yield several improvements to the overall course delivery. In order to achieve these improvements, we base a portion of our development on knowledge gained from the experience of other researchers in the area of control education who have looked at the usefulness of including multimedia tools to enhance learning. For example, the work of [1] describes how one may enhance the visualisation process and understanding of the theory of control engineering through provision of variety of online $\left(\mathrm{Matlab}^{1}\right)$ tutorials/simulations. The work of [2] allows access to a virtual control laboratory to perform a variety of experiments which help to develop an awareness of the application of the theory to practice. We make use of ideas such as HTML based tutorials and the provision of software simulations of practical systems to illustrate control theory concepts.

Section 2 of this paper describes the lecture component of the course, showing how the WWW may be advantageously used to disseminate lecture material, tutorial notes, assignments and course updates. Section 3 describes the laboratory component of the course, in which a DC servo motor setup is used throughout a variety of experiments over the course of a semester.

\footnotetext{
${ }^{1}$ Matlab is a registered trademark of The MathWorks, Inc.
} 
Section 4 focuses on the final course project, and serves to illustrate how combining the Web with simulation tools helps to solidify control engineering design concepts. The paper concludes with an evaluation of both the development and implementation of the course.

\section{The Undergraduate Course in Linear Process Control}

The lecture component of the course deals with the mathematical modeling, analysis, and design of linear, time-invariant systems and controllers. The curriculum includes a comprehensive treatment of the basic properties of feedback control, the design of the classic threeterm (PID) controller, controller tuning, stability analysis, and frequency response compensation techniques.

Underpinning these topics are complex mathematical foundations, which we find difficult to communicate properly to students when one is limited to classic teaching methods. Furthermore, teaching the design aspects of the course inherently requires an iterative process which can be greatly enhanced by introducing multimedia tools to illustrate the results. For example, consider the topic of pole/zero locations, and their subsequent effect on system behaviour. At best, using a classic approach, one is resigned to the tedious task of sketching diagrams of the system response, typically to a step input, for various pole locations. Contrast this to the much more illustrative means provided by [3] in which a Java applet allows one to place the singularities anywhere in the complex plane, and immediately see the results, or to a Matlab "movie", showing a transition from a pole-zero map to a frequency response plot, [4]. Both of these visualisations are embedded in the HTML pages and can be accessed on the World Wide Web with nothing more than a browser as a tool. Another improvement is to use software with a user friendly interface, such as a Matlab GUI applet, or Simulink ${ }^{2}$ simulation, allowing instant modifications (i.e. interactivity) without programming, such as in [5].

\subsection{Incorporating Multimedia Components in Lectures}

In the classroom, hyperlinked textual information is combined with simulations and multimedia components including video clips and screen captures, which readily provide a visualisation of the theory, and can illustrate real-life implementations thereof. Online availability of a complete set of lecture notes, containing embedded examples and tutorials frees the students to actively participate in the lecture process. The notes are accessed at a password-protected Web site and downloaded through FTP. We are also investigating the option of providing students with a CD-ROM copy of the lectures and tutorials in the next offering of the course, and with the WWW used to update and add to the information.

${ }^{2}$ Simulink is a registered trademark of The MathWorks, Inc.
The initial "big picture" lecture, in which we attempt to provide the students with a flavour of the course, is a good example of the effective use of multimedia. In the absence of any theoretical frame of reference and yet to be defined "jargon", we use video clips embedded in the HTML lecture notes to provide a visual, intuitive understanding of what is process control. We demonstrate the differences between effective and poor control while illustrating concepts of stability and instability, and transient system behaviour via a practical system setup. Using multimedia over a "real-life" demonstration has the advantage of being portable, faster, and allows us to use a variety of examples. For our course we created QuickTime movies of an inverted pendulum setup and two different servo positioning systems.

Outside of the classroom, implementing interactivity through the simulation software is a bit more complicated, since students must have access not only to tutorials through a browser, but they also require the Student Editions of Matlab and Simulink to be simultaneously running on their PC. Our current Web-based modules provide both lecture and lab tutorials that contain hypertext links to Simulink files which can be downloaded, executed and modified as required. Matlab commands are displayed in HTML documents, and can be cut and pasted into the software command window. Tutorial files also include captures of screen activity, and are effective in illustrating the use of Simulink drag-anddrop icons and in demonstrating simulation results. Tutorial screen captures, encoded using Lotus ScreenCam, are converted into animated GIFs to avoid a need for browser plug-ins and helper applications.

\section{Practical Servomotor Project}

Theory in the course is reinforced by hands-on experimental work and computer simulations using Matlab and Simulink. Lab projects utilize a positioning servo setup, shown in Figure 1.

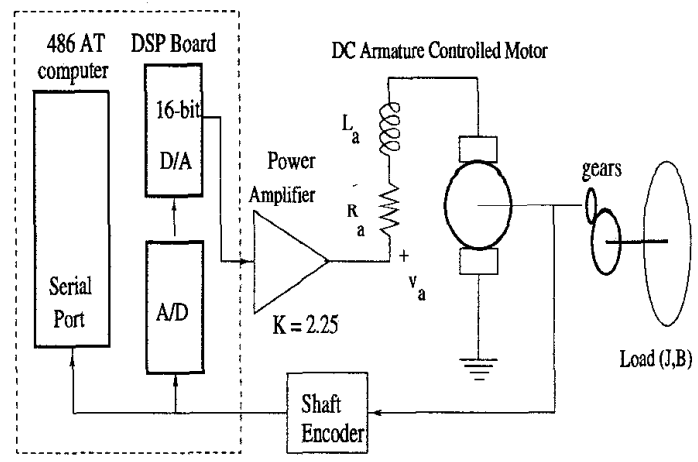

Figure 1: Block Diagram of the Servo Module.

The servo implements a high precision geared DC motor, equipped with an HP incremental optical en- 
coder, and a Motorola digital signal processing board with 16 bit $\mathrm{D} / \mathrm{A}$ and $\mathrm{A} / \mathrm{D}$ converters. The servo, with a pointer as its load, is enclosed in a rugged casing. The software, developed in-house, allows students to implement real-time PID or lead-lag control of the servo through a simple menu-driven interface. A choice of the positioning servo as an example of a process to be controlled has several advantages. It gives students insight into a range of real life phenomena such as saturation, gear backlash, and nonlinear friction, while providing reliable laboratory setups for students to obtain repeatable measurements throughout the semester. The setups are flexible and allow for a future expansion to implement more challenging control problems. The visual aspect of a functioning control system should not be overlooked as well; a servo system, be it a cutting tool, or an inverted pendulum, is more effective in illustrating basic concepts to novices than say, a temperature control system, even though it may be of the same difficulty on a conceptual level.

\subsection{Lab Description}

Experiments performed in the lab are tied closely to the theory covered in lectures and progress from system identification, to a simple controller design and implementation, to a 2-dimensional trajectory control problem. The lab format is 3 hours/week over the 13 week course. In the first of three experiments a model of the servo is derived using a classic approach based on step and frequency response measurements. The final model incorporates known nonlinearities and is verified using Simulink. In the second experiment an effective controller is designed and verified through simulations, then implemented in the physical setup. Unlike in many introductory courses in linear control, real life nonlinearities and resulting implementation implications are dealt with. For example, an anti-windup controller scheme is implemented, counteracting effects of saturation in the controller output, resulting from a limited range of the D/A converter (only \pm 1.4 volts). The final experiment is a Simulink simulation project described in Section 4.

\subsection{Incorporating Multimedia Components in Lab Instruction}

In a lab context, the Web is effectively used to disseminate project schedules, PostScript files of lab instructions, Matlab and Simulink files, and tutorials on software use. Students are introduced to the networked environment (Unix) and to Matlab in earlier courses in the second year of our curriculum, but are not initially familiar with the Matlab Control Toolbox package. While the purchase of Student Editions of both Matlab and Simulink software is recommended in the Control course, many students opt to rely exclusively on the network version available on campus. Some purchase just Matlab, as it is used in other courses. In either case, without access to manuals, their ability to attain proficiency in the control aspects of Matlab and in
Simulink is hindered. Since that proficiency is expected very early on in the course, online tutorials have the advantage of providing effective asynchronous delivery of the basic information and exercise material. Instructors and teaching assistants are relieved of fielding repetitive inquiries of the software basics.

Tutorials containing embedded simulations also prove to be an effective tool in delivering information about practical, but unfamiliar aspects of the servo module that fall outside the conventional linear control theory, such as how to identify and deal with DC offsets, D/A quantization errors and saturation, gear backlash and deadzone.

\section{Servomotor Driven X-Y Cutting Tool Simulation Project}

The final project in the course deals with an $x-y$ motion control problem. Currently a computer simulation, this project will eventually have a hardware implementation using a multichannel DSP board, two servornechanisms and an $\mathrm{x}-\mathrm{y}$ plotter as an analogy to a cutting tool. Simulink models of the problem is shown in Figures 2,3 , and 4 .

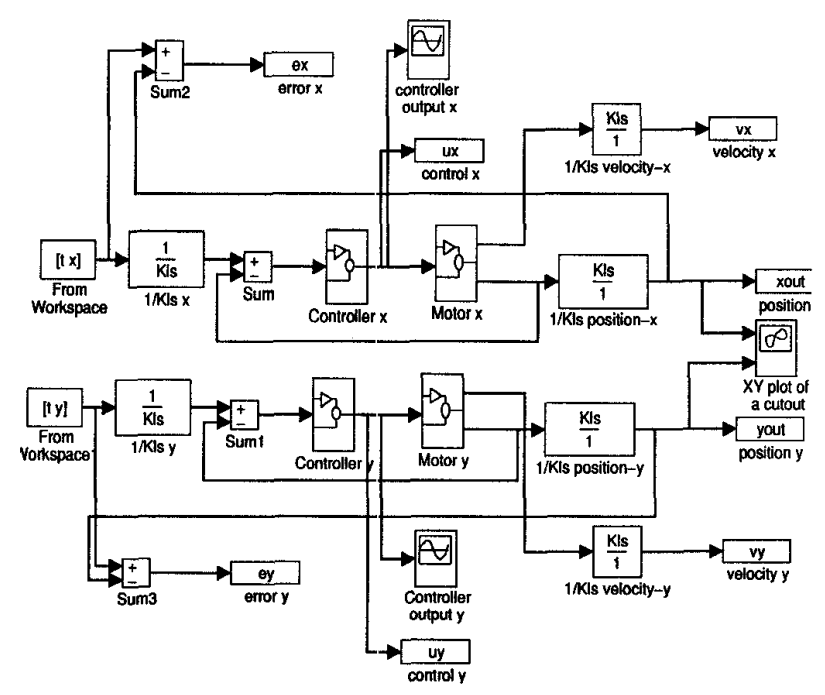

Figure 2: Simulink X-Y Axis Model of Cutting Tool Simulation.

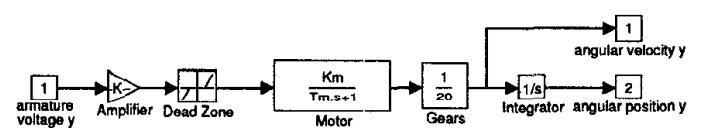

Figure 3: Simulink Model of Motor. 


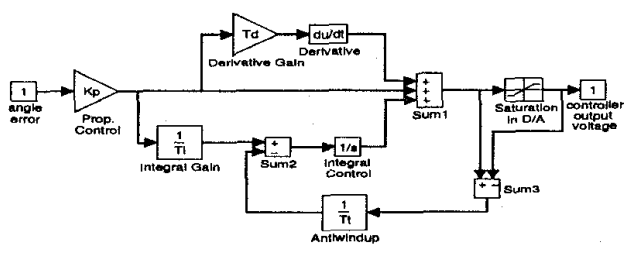

Figure 4: Simulink Model of PID Controller with Antiwindup.

The purpose of the project is to demonstrate the practical applications of control design, beyond meeting a set of specifications for standard inputs such as a step or a ramp signal. The project requirements are twofold: to plan a two-axis trajectory required to obtain a cutout of a specific shape (a rectangle and a circle), and to design and implement, through simulation, an effective control scheme to meet a set of requirements.

The requirements include restrictions on the maximum dimensional error of the cutout, on signal saturation (no on-off control allowed), and on the order of the controller (limited to fifth). Students work in teams and are allowed to explore any viable control scheme (PI, PID, in combination with lead-lag, feed-forward, velocity feedback, etc.) that meets the requirements.

\subsection{Project Competition}

Students engage in a friendly competition, where the objective is to obtain the fastest cutout time, while meeting the control specifications. There is enough flexibility built into the implementation to encourage creativity, and to develop a "feel" for the "art of control engineering", while providing a sound test of skills acquired in the course. Interesting dynamics result from the fact that the course is common for students from two engineering options: electrical engineering and computer engineering. The students are required to submit their simulations electronically to the instructor for verification of results. The final lab sessions are devoted to group presentations. While all groups are required to submit a hard copy semi-formal project report, the format of the presentation is left intentionally open, and may incorporate overheads, or multimedia presentation of computer simulations. A question and answer period follows. This aspect of the project provides the students with their first exposure to the rigours of reporting results in a coherent manner, both in a written and verbal form. This proves of value for the 4 th year thesis project.

\section{Conclusions}

By introducing multimedia into the classroom, the normally passive student who habitually sits, listens, and takes notes can be transformed into an enthusiastic student who takes an active role in learning. Outside of the classroom, students can access course materials from anywhere at anytime, both on and off campus. Web-based modules are dynamic, immediate, easy to update and maintain, and easy to access for both the students and the instructor. The networked environment allows a more effective course management through dissemination of information, on-line counseling, and increased communication. In our course we decided to forgo the creation of a newsgroup or a realtime chat room in favour of email only communications with and between students, in one-to-many and one-toone modes. We based this decision on the fact that email exchanges tend to be more task oriented, as well as asynchronous, while the newsgroups and chat rooms are more chaotic, and may lead to unproductive socializing. Chat rooms also require the synchronization of schedules, which is difficult to achieve for large groups of students. This has also been the experience of others [6].

Formal surveys on the impact of Web-based enhancements to the delivery of the controls course have not yet been applied. Multimedia-enhanced courses at Ryerson are still a relatively new experience, although they include participation in such initiatives as the Interactive Learning Connection - University Space Network (ILC USN), a project offering a Spacecraft Systems Design course in a collaboration between six universities and actively utilizing Internet and CD-ROM technologies. Ryerson Polytechnic University is well positioned to take leadership among Canadian undergraduate institutions in using new media to create stimulating interactive learning environments for students.

The vision statement of Ryerson focuses on providing the best possible applied undergraduate education through industry liaisons, an innovative curriculum, an investment in new technologies, and a Digital Media Projects Office [7], [8], dedicated to supporting faculty in the course development. A structured framework for technology enabled learning is being established. Its explicit goals are to focus on pedagogical strategies rather than specific technologies, to develop a methodology for assessing courseware, to test the framework with technology enabled courses, and to publish the results of testing and technology transfer. The controls course has been selected as one of the test beds for the framework, which will ensure quality of the development work and the methodologically sound course evaluation.

The anecdotal evidence gathered so far through informal discussions with students suggests that their enthusiasm and interest in the course, as well as the retention and comprehension of the concepts, increased. The average grade in the course went from $67 \%$ in the traditional course delivery in 1996 to $81 \%$ in the Webenhanced delivery in Winter, 1997. Incidentally, the dropout rate decreased to one, out of 97 students enrolled in the course, although it is premature to make 
any generalizations without a proper longitudinal study and prior to full implementation of all multimedia components of the course.

A Faculty Course Survey, which is a standard course evaluation tool, is applied each semester across the university to provide faculty with feedback.

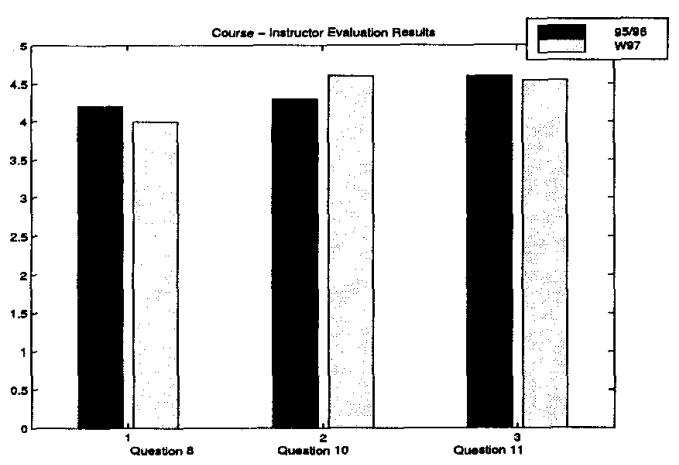

Figure 5: Results of Course Evaluations.

The 1997 results showed that the students' perception of the difficulty and the amount of course material decreased, when compared with the 1996 survey results (from 4.2 to 4.0 , out of 5), although there was no significant change in the actual course difficulty level or requirements. The overall evaluation of the instructors and the course increased slightly (from 4.3 to 4.6 , out of $5)$, consistent with our observations of increased enthusiasm and interest in the course. The Faculty Course Survey results are shown in Figure 5, where Question 8 was: "Rating of amount of course material", Question 10 was: "Overall, the faculty member was effective", and Question 11 was: "Overall, the course was worthwhile", with a scale 1 to 5 (Light - Heavy, and Disagree - Agree).

A review of the process required to create a technology enabled course shows that initially there is a great deal of work involved. Estimates of the ratio of the required preparation and development time in an initial offering of the technology enabled course to the conventional course are in the range of 20:1 [6]. Our own estimate is more conservative, as we had previously developed the conventional version of the course, and we possess a good deal of combined expertise both in the subject of the process control as well as in the area of multimedia applications. We further estimate that this ratio for subsequent course offerings should drop, but we feel that for the primary course developers it will still be in excess of the time required in the traditional approach to delivering the same course. However, there could be a substantial preparation time saving for a junior faculty or TA involved in delivering the developed course.

In conclusion, we feel that the results are encouraging, and despite the large outlay of resources, we intend to continue with this endeavour. It is our opinion that technology enabled courses, while not necessarily providing immediate savings of resources for the university, allow, when designed properly, a substantial, positive qualitative change in the way we educate engineering students.

\section{Acknowledgements}

The authors would like to express their gratitude to the Department of Electrical and Computer Engineering, Ryerson Polytechnic University, for their support of these advanced initiatives in course development. We would especially like to thank Mr. Jason Naughton who spent many hours in the design and development of the Servo Motor Modules.

\section{References}

[1] Bill Messner, Carnegie Mellon University, Dawn Tilbury, University of Michigan. Control Tutorials for MATLAB. http://www.engin.umich.edu/group/ctm/index.html

[2] Control Engineering Laboratory, RuhrUniversity Bochum. Virtual Control Lab. http://www.esr.ruhr-uni-bochum.de/ $\mathrm{vCLab} / \mathrm{main}$.htm1

[3] Systems Theory Institute, Braumschweig Technical University. Pole Zero Java Applet. http://www.nst.ing.tu-bs.de/schaukasten/ polezero/en_idx.htm.l

[4] Jeffrey B. Schodorf, Mark A. Yoder, James H. McClellan, "Using Multimedia to Teach the Theory of Digital Multimedia Signals", IEEE Transactions on Education, vol. 39, no. 3, pp 336-341, August 1996. http://www.ece.gatech.edu/research/DSP/courses/ ee2200/ee2200cd/ieee1995/Revision/pap296.htm

[5] Craig Ulmer, Georgia Institute of Technology. MATLAB Pole-Zero Graphical User Interface (GUI). http://www.ee.gatech.edu/research/DSP/courses/ ee2200/ee2200cd/ieee1995/Revision/Demos/Pez/ index.htm

[6] Carl Cuneo, National Director of the Network for the Evaluation of Education and Training Technologies, Education and the Internet: A Critical View, Invited Seminar, Ryerson Polytechnic University, March, 1998. http://socserv2.mcmaster.ca/srnet/evnet.htm

[7] Rogers Communications Centre, Ryerson Polytechnic University. http://www.rcc.ryerson.ca

[8] Digital Media Projects Office, Ryerson Polytechnic University. http://www.rcc.ryerson.ca/dmp/ 